\title{
Off-target challenge for base editor-mediated genome editing
}

\author{
Puping Liang • Junjiu Huang (D)
}

Received: 5 April 2019 / Accepted: 18 April 2019/Published online: 30 April 2019

(C) Springer Nature B.V. 2019

Genome editing is a revolutionary technology that holds immense promise for creating livestock or crops with improved traits and curing incurable inherited diseases by correcting genomic DNA mutation. The past decade has witnessed rapid developments of genome editing tools, such as zinc finger nuclease (ZFN), TALEN nuclease, and CRISPR/Cas nuclease. These programmable nucleases have been utilized to edit the genome by inducing DNA double-strand break (DSB), which will be then repaired by either non-homologous end joining (NHEJ) or homology directed repair (HDR). However, the efficiency of HDR is still low, and DSB poses a significant threat to genome stability, whose dysregulation might result in a serious disease like cancer.

To tackle these issues, new genome editing tools, named base editors, which could catalyze highly efficient base deamination-induced base transition without inducing DSB were developed (Gaudelli et al. 2017; Nishida et al. 2016; Komor et al. 2016). There are two types of base editors, cytidine base editor (CBE, C-to-T

P. Liang $\cdot$ J. Huang

Key Laboratory of Reproductive Medicine of Guangdong Province, The Third Affiliated Hospital of Guangzhou Medical University and School of Life Sciences of Sun Yat-sen University, Guangzhou 510000, China

\section{P. Liang $\cdot$ J. Huang $(\bowtie)$}

MOE Key Laboratory of Gene Function and Regulation, School of Life Sciences, Sun Yat-sen University, Guangzhou 510275, China

e-mail: hjunjiu@mail.sysu.edu.cn transition) and adenine base editor (ABE, A-to-G transition). CBE, composed of rAPOBEC1:nCas9:UGI effector and gRNA, harnesses rAPOBEC1 to deaminate cytidine (C) on the non-target strand and nCas9 (nickase Cas9) to cleave the target strand. ABE, composed of TADA:TADA*:nCas9 effector and gRNA, utilizes TADA to deaminate adenine (A) on the non-target strand and nCas9 to cleave the target strand. Without inducing DSB, such programmed "chemical surgery" on DNA was supposed to be more precise and efficient. Recently, both our group and Kim et al. developed in vitro assays (EndoV-seq and Digenome-seq) to investigate the genome-wide specificity of $\mathrm{CBE}$ and $\mathrm{ABE}$, which could detect the gRNA-dependent off-target of base editors. We found that $\mathrm{CBE}$ and $\mathrm{ABE}$ were much more specific than Cas9 nuclease (Kim et al. 2017a, 2019; Liang et al. 2019). However, both EndoV-seq and Digenome-seq might underestimate the off-target effects of base editors, because they could not detect offtarget deamination independent of gRNA recognition and nCas9 cleavage that might occur in vitro and in vivo (Kim et al. 2017a, 2019; Liang et al. 2019).

Recently, Zuo et al. and Jin et al. independently devised experiments to profile the genome-wide specificity of $\mathrm{CBE}, \mathrm{ABE}$, and Cas9 nuclease in mouse embryo or rice (Zuo et al. 2019; Jin et al. 2019). Previous attempts to detect genome-wide off-target effects of genome editing tools, especially for the single-nucleotide variations (SNVs), were hampered by genetic heterogeneity of individual organism, spontaneous mutation rate, the coverage of whole genome amplification, whole genome 
amplification errors, and sequencing errors. Zuo et al. eliminated genetic heterogeneity by using two genetically identical blastomeres in mouse 2-cell stage embryo (Zuo et al. 2019). The edited blastomere and its derivatives were labeled with tdTomato permanently $\left(\right.$ tdTomato $^{+}$), while the other unedited blastomere and its derivatives were tdTomato negative (tdTomato ${ }^{-}$). To get rid of low coverage and high errors associated with whole genome amplification, Zuo et al. smartly took advantage of embryogenesis to amplify the genome of the edited blastomere (Zuo et al. 2019). The edited 2-cell embryos were implanted into foster mother and harvested at embryonic day 14.5. Through this approach, they got enough edited $\left(\right.$ tdTomato $^{+}$) cells and unedited (tdTomato ${ }^{-}$) cells to extract genomic DNA for whole genome sequencing (WGS). Comparing the edited cells with unedited cells, they found that the CBE-edited mouse showed 20-fold more SNVs than the unedited control, while ABE and Cas9 nuclease groups displayed very rare SNVs (Zuo et al. 2019). Using rice model, Jin et al. found a similar phenomenon (Jin et al. 2019). They found that off-target SNVs caused by CBEs are independent of the gRNA, and even the high-fidelity CBE variant (HF1-BE3) induced similar number of SNVs comparable to the original CBE (Jin et al. 2019). Furthermore, SNVs are dominated by C-to- $\mathrm{T}$ conversions and enriched in actively transcribed region (Zuo et al. 2019; Jin et al. 2019). Collectively, these two studies demonstrated that CBE have significant off-target effects in vivo, causing a crisis for the application of CBE base editor, especially for its clinical translation. On the other side, their results and ours confirmed that $\mathrm{ABE}$ might be the most precise tool for genomic editing so far.

These two studies also attract a lot of attention to the mechanism underlying CBE off-target effects as well as how to improve the specificity of CBE. APOBEC1 protein per se could deaminate both DNA and RNA without the guidance of gRNA-nCas9 (Yang et al. 2014), while TADA protein could only deaminate DNA under the guidance of gRNA-nCas9 (Gaudelli et al. 2017). In addition, the local sequence context of the deaminated $\mathrm{C}$ showed a preference for $\mathrm{T}$ (thymine) at the $5^{\prime}$ end ( $5^{\prime}$-TC- $3^{\prime}$ ). In depth analysis of the sequence context of the 20 validated SNVs, we found that $\mathrm{T}$ is upstream the deaminated $\mathrm{C}$ at $17 \mathrm{SNVs}(17 / 20$, $85 \%$ ), in consistent with APOBEC1's preference for 5'-TC$3^{\prime}$ (Yang et al. 2014). In addition, ectopic expression of UGI (uracil-DNA glycosylase inhibitor) in human glioma U215 cell led to a 3-fold increase of the DNA mutation rate (Radany et al. 2000). These data clearly indicated that the inherent DNA-binding activity of APOBEC1 and overexpression of UGI resulted in CBE off-targets. Furthermore, these data highlight the need for a comprehensive study to elucidate the underlying mechanism of CBE off-target effects. And more efforts should be devoted to improving the specificity of CBE. There are many cytidine deaminases such as attenuated APOBEC1 mutants (YE1, YE2, EE, and YEE), APOBEC homologs, AID, and CDA1. It remains unknown whether other cytidine deaminases will cause similar off-target deamination (Kim et al. 2017b; Koblan et al. 2018). In addition, it might be feasible to reduce CBE off-target effects by delivering ribonucleoprotein instead of DNA or RNA, controlling CBE expression and duration by inducible system (Rees et al. 2017; Dow et al. 2015; Kleinjan et al. 2017). On the other hand, CBE off-target effects should be studied in a "real-world" preclinical application, such as human somatic cells.

In sum, although the CBE has obvious off-target effects, both in vitro and in vivo studies demonstrated that $\mathrm{ABE}$ is highly specific, paving the way for the clinical application of ABE (Liang et al. 2019; Kim et al. 2019; Zuo et al. 2019; Jin et al. 2019).

Funding information This work was supported by the National Key R\&D Program of China (2017YFC1001901), the National Natural Science Foundation (31601196), and the Guangzhou Science and Technology Project (201803010020 and 201707010085).

\section{References}

Dow LE, Fisher J, O'Rourke KP, Muley A, Kastenhuber ER, Livshits $\mathrm{G}$, et al. Inducible in vivo genome editing with CRISPR-Cas9. Nat Biotechnol. 2015;33:390-4.

Gaudelli NM, Komor AC, Rees HA, Packer MS, Badran AH, Bryson DI, et al. Programmable base editing of $A^{*} \mathrm{~T}$ to $\mathrm{G}^{*} \mathrm{C}$ in genomic DNA without DNA cleavage. Nature. 2017;551: 464-71.

Jin S, Zong Y, Gao Q, Zhu Z, Wang Y, Qin P, et al. Cytosine, but not adenine, base editors induce genome-wide off-target mutations in rice. Science. 2019:eaaw7166.

Kim D, Lim K, Kim ST, Yoon SH, Kim K, Ryu SM, et al. Genomewide target specificities of CRISPR RNA-guided programmable deaminases. Nat Biotechnol. 2017a;35:475-80.

Kim YB, Komor AC, Levy JM, Packer MS, Zhao KT, Liu DR. Increasing the genome-targeting scope and precision of base editing with engineered Cas9-cytidine deaminase fusions. Nat Biotechnol. 2017b;35:371-6.

Kim D, Kim DE, Lee G, Cho SI, Kim JS. Genome-wide target specificity of CRISPR RNA-guided adenine base editors. Nat Biotechnol. 2019;37:430-5.

Kleinjan DA, Wardrope C, Nga Sou S, Rosser SJ. Drug-tunable multidimensional synthetic gene control using inducible 
degron-tagged dCas9 effectors. Nat Commun. 2017;8(1191): 1191.

Koblan LW, Doman JL, Wilson C, Levy JM, Tay T, Newby GA, et al. Improving cytidine and adenine base editors by expression optimization and ancestral reconstruction. Nat Biotechnol. 2018;36:843-6.

Komor AC, Kim YB, Packer MS, Zuris JA, Liu DR. Programmable editing of a target base in genomic DNA without double-stranded DNA cleavage. Nature. 2016;533:420-4.

Liang P, Xie X, Zhi S, Sun H, Zhang X, Chen Y, et al. Genomewide profiling of adenine base editor specificity by EndoVseq. Nat Commun. 2019;10(67):67.

Nishida K, Arazoe T, Yachie N, Banno S, Kakimoto M, Tabata M, et al. Targeted nucleotide editing using hybrid prokaryotic and vertebrate adaptive immune systems. Science. 2016;353:aaf8729.

Radany EH, Dornfeld KJ, Sanderson RJ, Savage MK, Majumdar A, Seidman MM, et al. Increased spontaneous mutation frequency in human cells expressing the phage PBS2encoded inhibitor of uracil-DNA glycosylase. Mutat Res. 2000;461:41-58.

Rees HA, Komor AC, Yeh WH, Caetano-Lopes J, Warman M, Edge ASB, et al. Improving the DNA specificity and applicability of base editing through protein engineering and protein delivery. Nat Commun. 2017;8:15790.

Yang L, Grishin D, Wang G, Aach J, Zhang CZ, Chari R, et al. Targeted and genome-wide sequencing reveal single nucleotide variations impacting specificity of Cas 9 in human stem cells. Nat Commun. 2014;5(5507):5507.

Zuo E, Sun Y, Wei W, Yuan T, Ying W, Sun H, et al. Cytosine base editor generates substantial off-target single-nucleotide variants in mouse embryos. Science. 2019:eaav9973.

Publisher's note Springer Nature remains neutral with regard to jurisdictional claims in published maps and institutional affiliations. 